\title{
R. C. Baselt (ed.): Disposition of toxic drugs and chemicals in man, 10th edition
}

\author{
Biomedical Publications, Seal Beach, California (USA), 2014, hardbound, \\ ISBN: 978-0-9626523-9-4
}

\author{
Martin Hastedt
}

Accepted: 2 August 2014/Published online: 28 August 2014

(c) Springer Science+Business Media New York 2014

Disposition of Toxic Drugs and Chemicals in Man is one of the most noteworthy books in the field of pharmacology, pharmacy, forensic, and clinical toxicology. Recently, the 10th edition of this standard work has been published. The book includes monographs for more than 1,500 unique chemicals. For each substance data on body fluid concentrations in therapeutic settings or in case of intoxications, as well as data on occurrence and usage, metabolism and excretion, toxicity, analytical procedures and on the most important pharmacokinetic parameters is given.

For the 10th edition, more than 270 drugs or chemicals from different scopes were added. The fields of application of these substances vary from new pharmaceuticals, antidotes, contrast agents, essential elements, and plant alkaloids, to recreational drugs. Disposition of Toxic Drugs and Chemicals in Man stands out due to its actuality. Many of the newly added pharmaceuticals arose on the market within the last few years. Beside the addition of new substances there were many updates for the preexisting monographs. Another feature that highlights its actuality is the addendum that can be found prior to the index. It includes recently developed drugs or drugs of abuse. Examples are 25B-NBOMe a partial 5-HT2A-receptor agonist and the cathinone derivative buphedrone. In the 9th edition the prologue "Guidelines for Interpretation of Analytical Toxicology Results" was implemented. With the actual 10th edition this section has been updated and expanded especially regarding fundamental pharmacokinetic guidelines. At the top of every monograph half life, volume of distribution, bioavailability, $\mathrm{pKa}$, blood/plasma ratio, and the chemical structure are reported. Information on Chemical Abstracts Service (CAS) registry number, molecular weight, and the sum formula was added for the 10th edition and completes the table of the most important substance data.

One point of criticism is that there is still no electronic version of this edition. An electronic version could make inquiries much easier-for example the search for keywords. References could be electronically linked, and information could be downloaded on mobile gadgets. Nevertheless it is positive that the 10th edition of Disposition of Toxic Drugs and Chemicals in Man is still in a single volume format and although there is a total of 2,250 pages, the dimensions are, in relation to the extent of the book, rather compact.

The quality of the descriptions and monographs is excellent. Information on the disposition of the chemicals and drugs, concentrations in body fluids and tissues, and the metabolic pathway is well condensed to the most important facts. Disposition of Toxic Drugs and Chemicals in Man has become an essential reference book in many laboratories that deal with clinical or forensic cases of poisoning. The purchase of this book can be recommended, especially for all clinical and forensic toxicologists.
M. Hastedt $(\bowtie)$

Institute of Legal Medicine and Forensic Sciences,

Charité - Universitätsmedizin Berlin, Turmstraße 21 (Haus N),

10059 Berlin, Germany

e-mail: martin.hastedt@charite.de 